\title{
Supplemental Information for: \\ Improving the Environmental and Economic Viability of U.S. Oil Shale via Waste-to- Byproduct Conversion of Semicoke to Sorbents
}

\author{
Ami Vyas ${ }^{1}$, Junjie Xue ${ }^{2,3}$, Jillian L. Goldfarb ${ }^{2,4^{*}}$ \\ 1. Department of Electrical and Computer Engineering, Boston University, 8 St. Mary's Street, Boston MA 02215 \\ 2. Department of Mechanical Engineering, Boston University, 110 Cummington Mall, Boston MA 02215 \\ 3. College of Engineering, China Agricultural University, Beijing 100083, People's Republic of China \\ 4. Division of Materials Science and Engineering, Boston University, 15 St. Mary's Street, Brookline MA 02446, \\ United States
}

\footnotetext{
* To whom correspondence should be addressed: JillianLGoldfarb@gmail.com; jilliang@bu.edu; (617) 3533883 (tel); (617) 3535866 (fax)
} 
Table S1. Activation energies of oxidation for raw, pyrolyzed and activated semicokes calculated by the Distributed Activation Energy Model (Raw at $X=0.75$ and pyrolyzed at $X=0.30$ omitted data as $\mathrm{R}^{2}<0.90$ )

\begin{tabular}{|c|c|c|c|c|}
\hline $\begin{array}{l}\text { Mass } \\
\text { Fraction } \\
\text { Converted }\end{array}$ & $\mathrm{E}(\mathrm{kJ} / \mathrm{mol})$ & $\mathrm{A}\left(\mathrm{s}^{-1}\right)$ & $\mathrm{E}(\mathrm{kJ} / \mathrm{mol})$ & $\mathrm{A}\left(\mathrm{s}^{-1}\right)$ \\
\hline & \multicolumn{2}{|c|}{ Raw Oil Shale } & \multicolumn{2}{|c|}{ Pyrolyzed Oil Shale } \\
\hline 0.10 & $98.3 \pm 13.0$ & $1.50 \mathrm{E}+04 \pm 1.31 \mathrm{E}+01$ & $92.5 \pm 12.9$ & $6.40 \mathrm{E}+02 \pm 9.94 \mathrm{E}+00$ \\
\hline 0.15 & $92.1 \pm 13.2$ & $2.32 \mathrm{E}+03 \pm 1.25 \mathrm{E}+01$ & $89.9 \pm 10.3$ & $2.73 \mathrm{E}+02 \pm 6.04 \mathrm{E}+00$ \\
\hline 0.20 & $79.1 \pm 12.8$ & $1.17 \mathrm{E}+02 \pm 1.08 \mathrm{E}+01$ & $88.9 \pm 9.4$ & $1.60 \mathrm{E}+02 \pm 4.96 \mathrm{E}+00$ \\
\hline 0.25 & $67.5 \pm 14.5$ & $9.08 \mathrm{E}+00 \pm 1.39 \mathrm{E}+01$ & $85.9 \pm 9.2$ & $6.31 \mathrm{E}+01 \pm 4.62 \mathrm{E}+00$ \\
\hline 0.30 & $61.1 \pm 15.5$ & $2.05 \mathrm{E}+00 \pm 1.53 \mathrm{E}+01$ & & \\
\hline 0.35 & $57.6 \pm 15.8$ & $8.64 \mathrm{E}-01 \pm 1.54 \mathrm{E}+01$ & $265.0 \pm 29.8$ & $5.55 \mathrm{E}+10 \pm 5.35 \mathrm{E}+01$ \\
\hline 0.40 & $53.8 \pm 16.6$ & $3.65 \mathrm{E}-01 \pm 1.69 \mathrm{E}+01$ & $240.1 \pm 16.6$ & $9.07 \mathrm{E}+08 \pm 8.66 \mathrm{E}+00$ \\
\hline 0.45 & $50.4 \pm 17.0$ & $1.74 \mathrm{E}-01 \pm 1.71 \mathrm{E}+01$ & $236.2 \pm 10.3$ & $3.43 \mathrm{E}+08 \pm 3.78 \mathrm{E}+00$ \\
\hline 0.50 & $46.5 \pm 16.6$ & $7.70 \mathrm{E}-02 \pm 1.54 \mathrm{E}+01$ & $221.4 \pm 10.1$ & $3.61 \mathrm{E}+07 \pm 3.59 \mathrm{E}+00$ \\
\hline 0.55 & $44.0 \pm 15.8$ & $4.45 \mathrm{E}-02 \pm 1.71 \mathrm{E}+01$ & $219.5 \pm 8.5$ & $2.19 \mathrm{E}+07 \pm 2.90 \mathrm{E}+00$ \\
\hline 0.60 & $42.6 \pm 15.2$ & $3.04 \mathrm{E}-02 \pm 1.54 \mathrm{E}+01$ & $216.8 \pm 7.5$ & $1.24 \mathrm{E}+07 \pm 2.56 \mathrm{E}+00$ \\
\hline 0.65 & $43.7 \pm 15.3$ & $3.00 \mathrm{E}-02 \pm 1.29 \mathrm{E}+01$ & $216.1 \pm 6.9$ & $9.32 \mathrm{E}+06 \pm 2.35 \mathrm{E}+00$ \\
\hline 0.70 & $117.0 \pm 44.6$ & $6.93 \mathrm{E}+02 \pm 1.13 \mathrm{E}+01$ & $214.6 \pm 2.5$ & $6.54 \mathrm{E}+06 \pm 1.35 \mathrm{E}+00$ \\
\hline 0.75 & & & $211.4 \pm 1.6$ & $3.77 \mathrm{E}+06 \pm 1.21 \mathrm{E}+00$ \\
\hline 0.80 & $302.7 \pm 38.1$ & $1.45 \mathrm{E}+12 \pm 6.53 \mathrm{E}+02$ & $208.4 \pm 2.9$ & $2.26 \mathrm{E}+06 \pm 1.42 \mathrm{E}+00$ \\
\hline 0.85 & $246.4 \pm 30.2$ & $4.10 \mathrm{E}+08 \pm 7.06 \mathrm{E}+02$ & $209.0 \pm 1.4$ & $2.12 \mathrm{E}+06 \pm 1.19 \mathrm{E}+00$ \\
\hline 0.90 & $205.8 \pm 16.7$ & $1.59 \mathrm{E}+06 \pm 1.30 \mathrm{E}+02$ & $207.8 \pm 1.8$ & $1.59 \mathrm{E}+06 \pm 1.24 \mathrm{E}+00$ \\
\hline \multirow[t]{2}{*}{ Average } & $100.5 \pm 9.5$ & $9.05 E+10 \pm 2.26 E+02$ & $189.0 \pm 7.1$ & $3.55 E+09 \pm 1.27 E+01$ \\
\hline & \multicolumn{2}{|c|}{ HCl Activated } & \multicolumn{2}{|c|}{ KOH Activated } \\
\hline 0.10 & $126.6 \pm 12.8$ & $1.49 \mathrm{E}+05 \pm 9.29 \mathrm{E}+00$ & $100.4 \pm 12.4$ & $2.00 \mathrm{E}+03 \pm 8.81 \mathrm{E}+00$ \\
\hline 0.15 & $126.6 \pm 7.5$ & $1.11 \mathrm{E}+05 \pm 3.65 \mathrm{E}+00$ & $97.0 \pm 10.7$ & $7.20 \mathrm{E}+02 \pm 6.28 \mathrm{E}+00$ \\
\hline 0.20 & $128.2 \pm 3.3$ & $1.19 \mathrm{E}+05 \pm 1.75 \mathrm{E}+00$ & $96.6 \pm 9.7$ & $4.89 \mathrm{E}+02 \pm 5.18 \mathrm{E}+00$ \\
\hline 0.25 & $133.2 \pm 0.6$ & $2.44 \mathrm{E}+05 \pm 1.10 \mathrm{E}+00$ & $96.0 \pm 10.9$ & $3.13 \mathrm{E}+02 \pm 6.13 \mathrm{E}+00$ \\
\hline 0.30 & $135.7 \pm 2.0$ & $3.28 \mathrm{E}+05 \pm 1.40 \mathrm{E}+00$ & $103.6 \pm 13.8$ & $5.96 \mathrm{E}+02 \pm 9.12 \mathrm{E}+00$ \\
\hline 0.35 & $133.0 \pm 2.2$ & $1.83 \mathrm{E}+05 \pm 1.45 \mathrm{E}+00$ & $268.8 \pm 126.5$ & $4.80 \mathrm{E}+11 \pm 4.48 \mathrm{E}+07$ \\
\hline 0.40 & $129.4 \pm 0.1$ & $8.95 \mathrm{E}+04 \pm 1.02 \mathrm{E}+00$ & $248.7 \pm 50.1$ & $3.90 \mathrm{E}+09 \pm 7.30 \mathrm{E}+02$ \\
\hline 0.45 & $127.9 \pm 0.4$ & $6.43 \mathrm{E}+04 \pm 1.08 \mathrm{E}+00$ & $236.4 \pm 42.6$ & $3.82 \mathrm{E}+08 \pm 2.43 \mathrm{E}+02$ \\
\hline 0.50 & $122.3 \pm 1.6$ & $2.32 \mathrm{E}+04 \pm 1.31 \mathrm{E}+00$ & $222.6 \pm 44.8$ & $4.26 \mathrm{E}+07 \pm 1.09 \mathrm{E}+02$ \\
\hline 0.55 & $120.6 \pm 2.2$ & $1.61 \mathrm{E}+04 \pm 1.44 \mathrm{E}+00$ & $215.2 \pm 43.8$ & $1.24 \mathrm{E}+07 \pm 2.48 \mathrm{E}+02$ \\
\hline 0.60 & $115.7 \pm 3.9$ & $6.66 \mathrm{E}+03 \pm 1.89 \mathrm{E}+00$ & $209.0 \pm 44.4$ & $4.52 \mathrm{E}+06 \pm 2.55 \mathrm{E}+02$ \\
\hline 0.65 & $111.8 \pm 4.5$ & $3.22 \mathrm{E}+03 \pm 2.08 \mathrm{E}+00$ & $210.8 \pm 42.7$ & $4.64 \mathrm{E}+06 \pm 1.98 \mathrm{E}+02$ \\
\hline 0.70 & $110.0 \pm 3.6$ & $2.25 \mathrm{E}+03 \pm 1.79 \mathrm{E}+00$ & $207.0 \pm 43.1$ & $2.43 \mathrm{E}+06 \pm 2.02 \mathrm{E}+02$ \\
\hline 0.75 & $107.0 \pm 4.4$ & $1.27 \mathrm{E}+03 \pm 2.06 \mathrm{E}+00$ & $202.3 \pm 42.0$ & $1.15 \mathrm{E}+06 \pm 1.70 \mathrm{E}+02$ \\
\hline 0.80 & $103.0 \pm 4.8$ & $6.11 \mathrm{E}+02 \pm 2.16 \mathrm{E}+00$ & $204.7 \pm 41.8$ & $1.35 \mathrm{E}+06 \pm 1.63 \mathrm{E}+02$ \\
\hline 0.85 & $100.6 \pm 4.1$ & $3.77 \mathrm{E}+02 \pm 1.94 \mathrm{E}+00$ & $203.0 \pm 40.5$ & $9.51 \mathrm{E}+05 \pm 1.35 \mathrm{E}+02$ \\
\hline 0.90 & $97.8 \pm 0.8$ & $2.03 \mathrm{E}+02 \pm 1.14 \mathrm{E}+00$ & $200.6 \pm 41.1$ & $6.20 \mathrm{E}+05 \pm 1.41 \mathrm{E}+02$ \\
\hline \multirow[t]{2}{*}{ Average } & $119.4 \pm 3.1$ & $7.89 E+04 \pm 1.94 E+00$ & $183.7 \pm 27.1$ & $2.85 E+10 \pm 1.09 E+07$ \\
\hline & \multicolumn{2}{|c|}{ HCI+KOH Activated } & \multicolumn{2}{|c|}{ KOH+HCl Activated } \\
\hline 0.10 & $125.4 \pm 14.9$ & $1.16 \mathrm{E}+05 \pm 1.34 \mathrm{E}+01$ & $145.6 \pm 13.6$ & $9.45 \mathrm{E}+06 \pm 1.15 \mathrm{E}+01$ \\
\hline 0.15 & $131.9 \pm 10.4$ & $2.72 \mathrm{E}+05 \pm 5.98 \mathrm{E}+00$ & $147.4 \pm 1.5$ & $8.97 \mathrm{E}+06 \pm 1.30 \mathrm{E}+00$ \\
\hline 0.20 & $133.0 \pm 15.3$ & $2.69 \mathrm{E}+05 \pm 1.35 \mathrm{E}+01$ & $154.6 \pm 10.5$ & $2.47 \mathrm{E}+07 \pm 6.34 \mathrm{E}+00$ \\
\hline 0.25 & $127.7 \pm 25.3$ & $9.53 \mathrm{E}+04 \pm 7.25 \mathrm{E}+01$ & $156.0 \pm 15.1$ & $2.58 \mathrm{E}+07 \pm 1.38 \mathrm{E}+01$ \\
\hline 0.30 & $120.7 \pm 30.4$ & $2.61 \mathrm{E}+04 \pm 1.67 \mathrm{E}+02$ & $147.0 \pm 9.8$ & $4.47 \mathrm{E}+06 \pm 5.42 \mathrm{E}+00$ \\
\hline 0.35 & $116.3 \pm 31.4$ & $1.11 \mathrm{E}+04 \pm 1.93 \mathrm{E}+02$ & $140.8 \pm 5.7$ & $1.30 \mathrm{E}+06 \pm 2.67 \mathrm{E}+00$ \\
\hline 0.40 & $110.5 \pm 29.5$ & $3.78 \mathrm{E}+03 \pm 1.38 \mathrm{E}+02$ & $136.1 \pm 4.7$ & $5.11 \mathrm{E}+05 \pm 2.22 \mathrm{E}+00$ \\
\hline 0.45 & $106.0 \pm 24.6$ & $1.60 \mathrm{E}+03 \pm 5.92 \mathrm{E}+01$ & $128.4 \pm 1.8$ & $1.21 \mathrm{E}+05 \pm 1.35 \mathrm{E}+00$ \\
\hline 0.50 & $103.4 \pm 21.1$ & $9.58 \mathrm{E}+02 \pm 3.24 \mathrm{E}+01$ & $123.5 \pm 1.6$ & $4.74 \mathrm{E}+04 \pm 1.31 \mathrm{E}+00$ \\
\hline 0.55 & $98.0 \pm 18.8$ & $3.61 \mathrm{E}+02 \pm 2.20 \mathrm{E}+01$ & $116.1 \pm 5.6$ & $1.22 \mathrm{E}+04 \pm 2.58 \mathrm{E}+00$ \\
\hline 0.60 & $95.6 \pm 16.8$ & $2.23 \mathrm{E}+02 \pm 1.55 \mathrm{E}+01$ & $111.7 \pm 6.2$ & $5.33 \mathrm{E}+03 \pm 2.81 \mathrm{E}+00$ \\
\hline 0.65 & $91.1 \pm 15.1$ & $9.88 \mathrm{E}+01 \pm 1.04 \mathrm{E}+01$ & $105.9 \pm 6.9$ & $1.84 \mathrm{E}+03 \pm 3.19 \mathrm{E}+00$ \\
\hline 0.70 & $87.5 \pm 13.2$ & $5.10 \mathrm{E}+01 \pm 8.41 \mathrm{E}+00$ & $100.8 \pm 7.5$ & $7.16 \mathrm{E}+02 \pm 3.51 \mathrm{E}+00$ \\
\hline 0.75 & $86.2 \pm 11.5$ & $3.86 \mathrm{E}+01 \pm 6.38 \mathrm{E}+00$ & $97.0 \pm 7.7$ & $3.52 \mathrm{E}+02 \pm 3.55 \mathrm{E}+00$ \\
\hline 0.80 & $82.5 \pm 10.5$ & $1.95 \mathrm{E}+01 \pm 5.41 \mathrm{E}+00$ & $93.1 \pm 8.4$ & $1.65 \mathrm{E}+02 \pm 4.00 \mathrm{E}+00$ \\
\hline 0.85 & $80.4 \pm 9.3$ & $1.27 \mathrm{E}+01 \pm 4.40 \mathrm{E}+00$ & $91.1 \pm 8.1$ & $1.06 \mathrm{E}+02 \pm 3.76 \mathrm{E}+00$ \\
\hline 0.90 & $79.0 \pm 6.9$ & $9.14 \mathrm{E}+00 \pm 3.00 \mathrm{E}+00$ & $91.6 \pm 4.7$ & $8.93 \mathrm{E}+01 \pm 2.13 \mathrm{E}+00$ \\
\hline Average & $104.4 \pm 7.8$ & $4.68 E+04 \pm 6.15 E+01$ & $122.7 \pm 3.8$ & $4.43 E+06 \pm 3.48 E+00$ \\
\hline
\end{tabular}




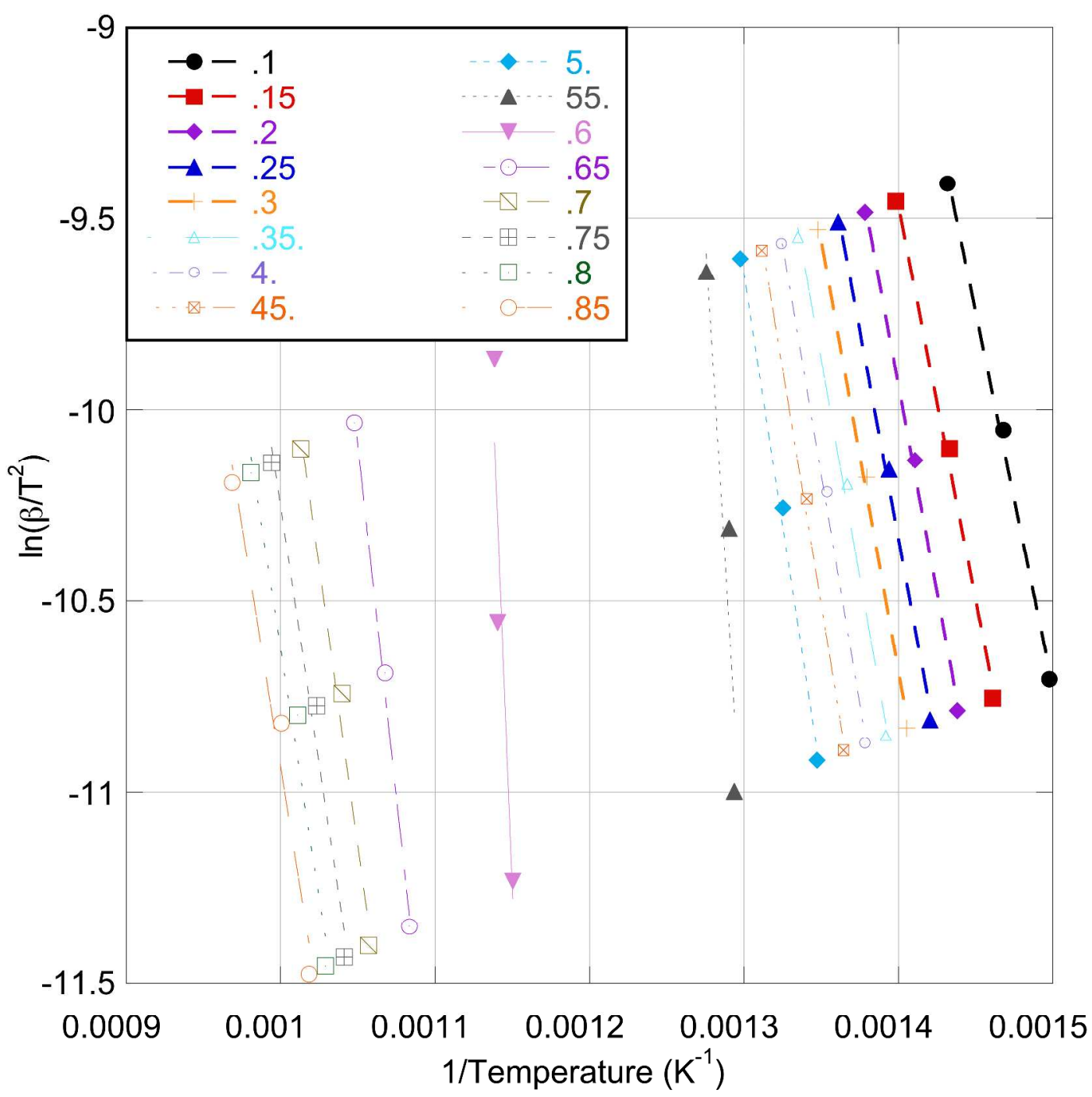

Figure S1. Isoconversional plot for activation energy of pyrolysis of raw White River Mine oil shale 


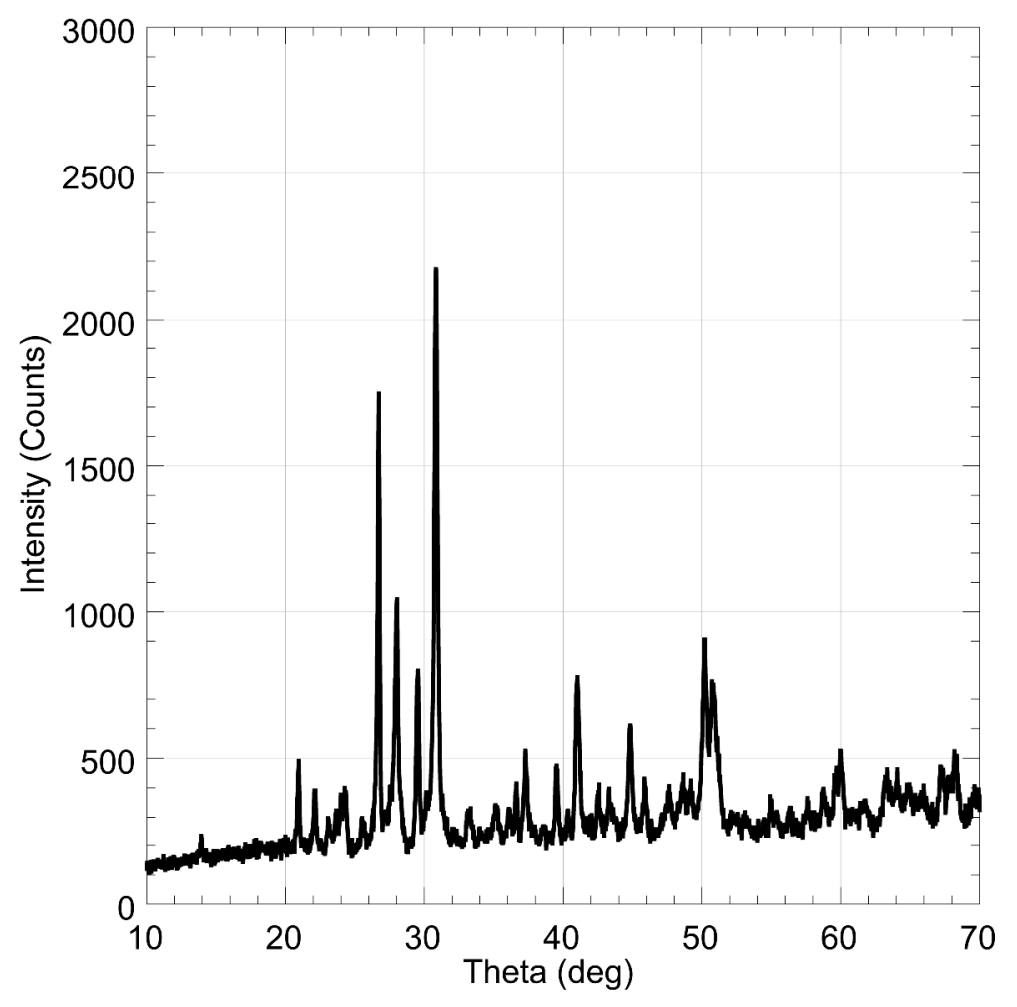

a. Raw WROS

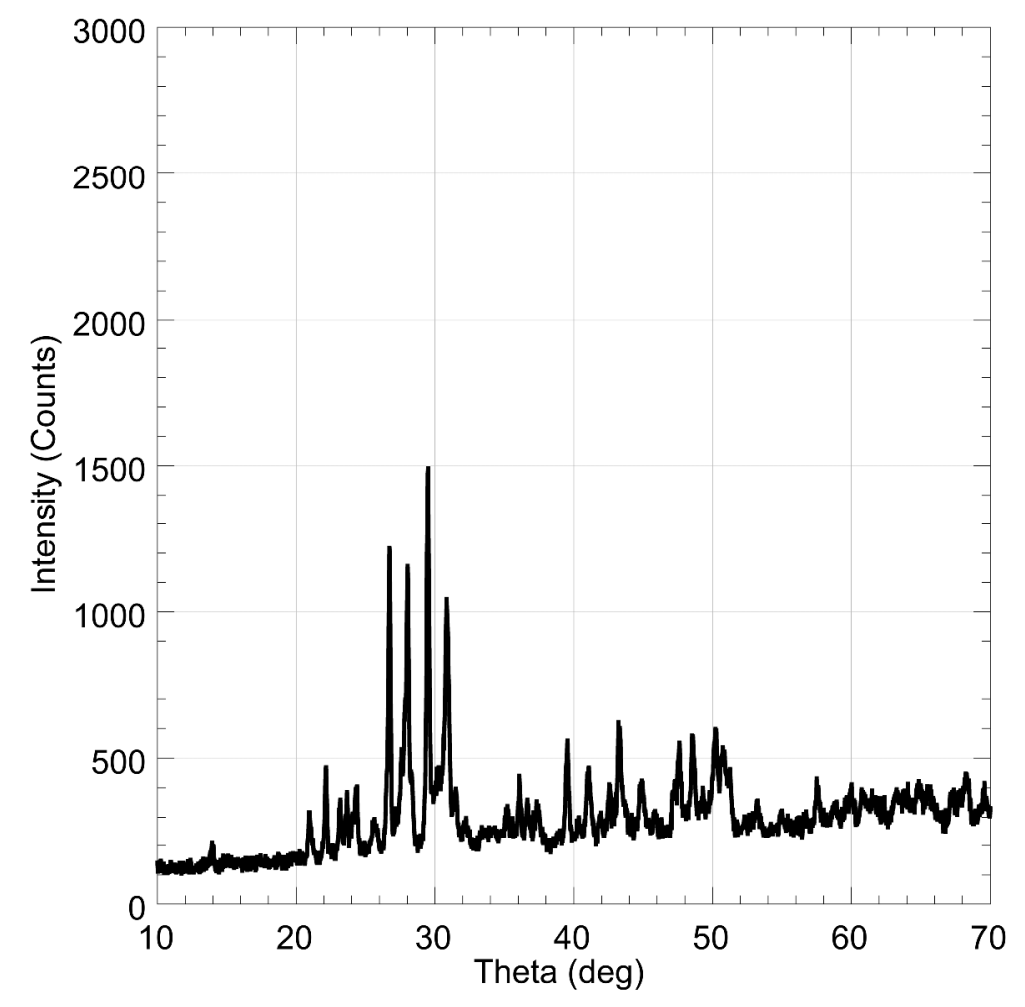

b. Semicoke 

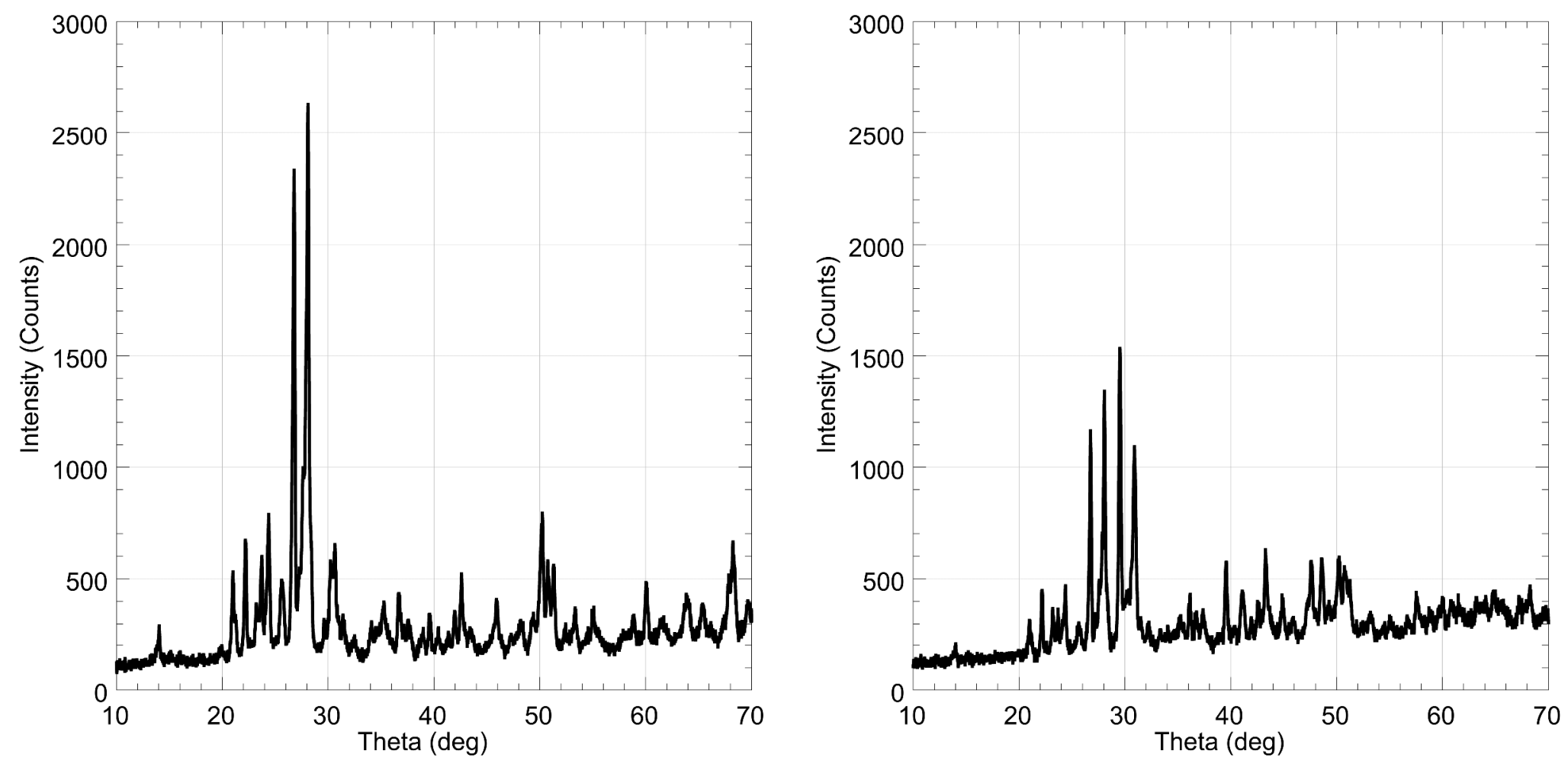

c. $\mathrm{HCl}$ Activated

d. $\mathrm{KOH}$ Activated 

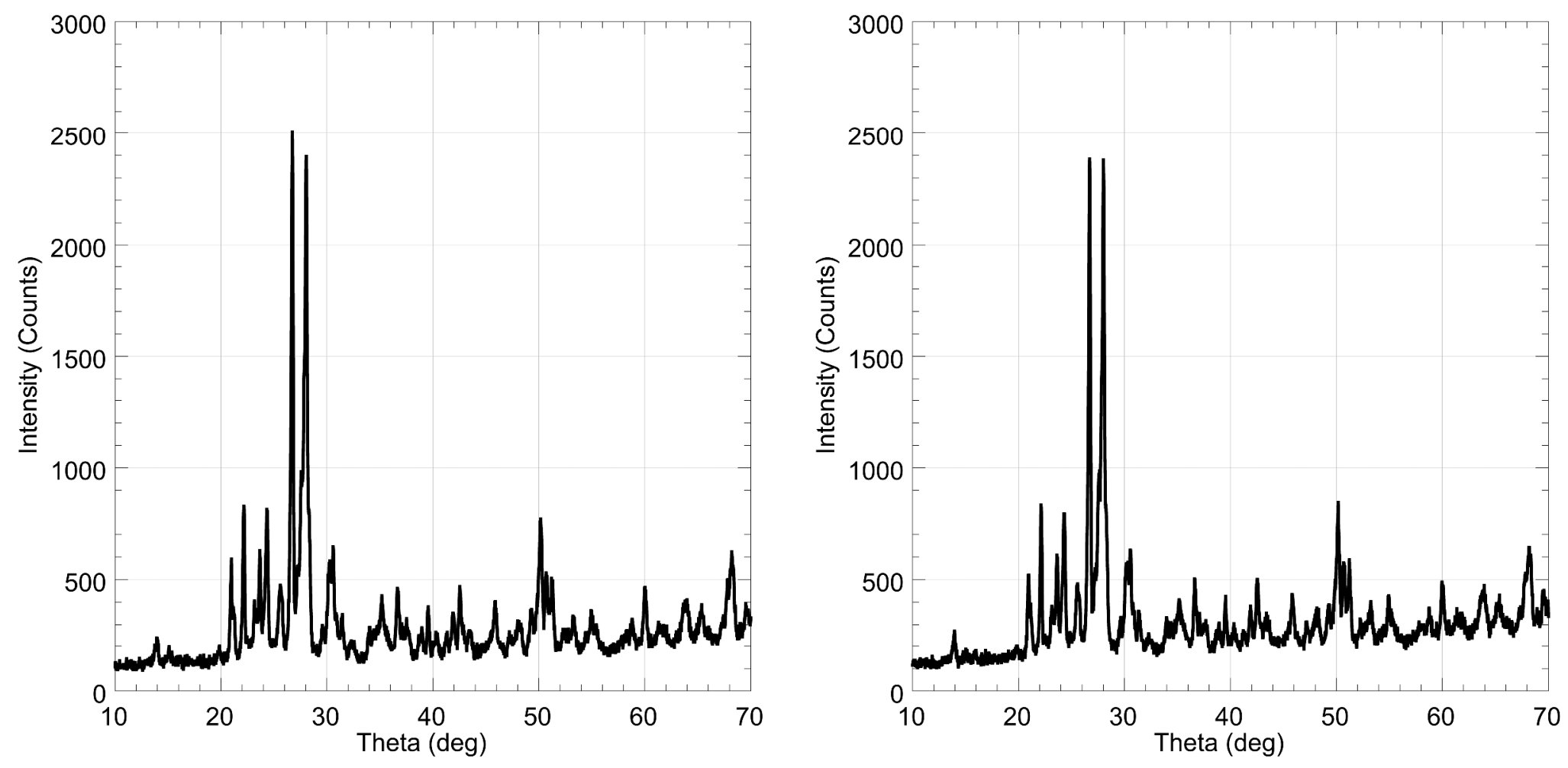

e. $\mathrm{HCl}+\mathrm{KOH}$ Activated

f. $\mathrm{KOH}+\mathrm{HCl}$ Activated

Figure S2. XRD data for raw, semicoke and activated semicoke samples 


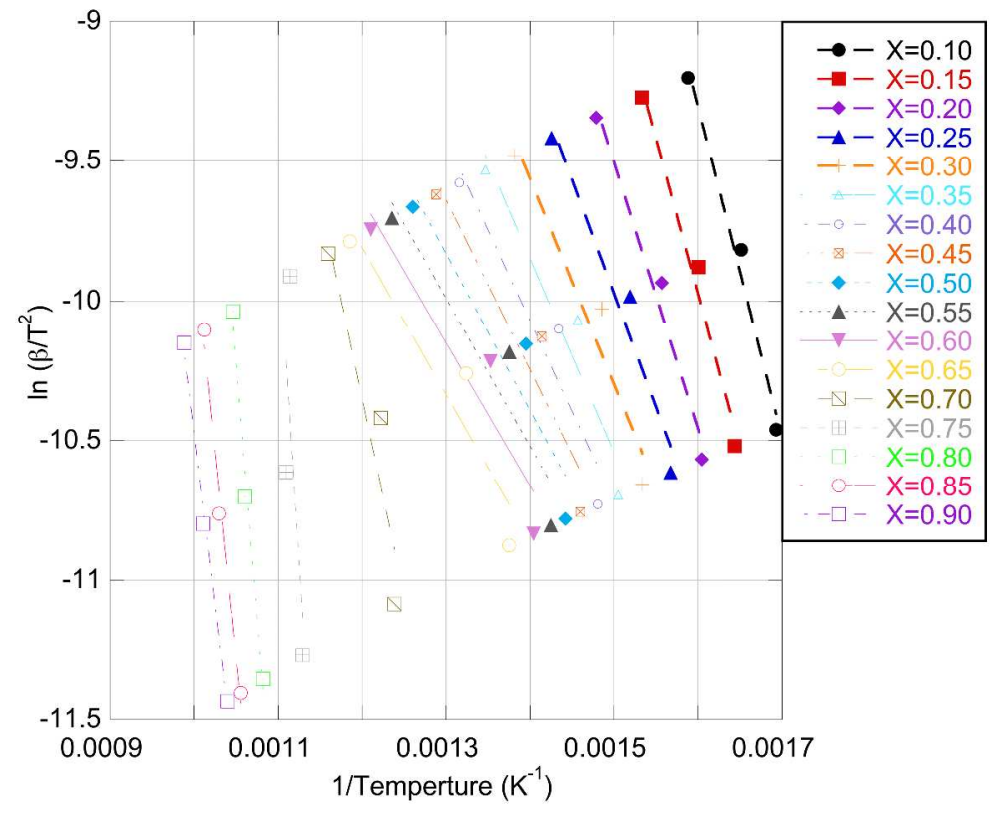

a. Raw WROS Oil Shale

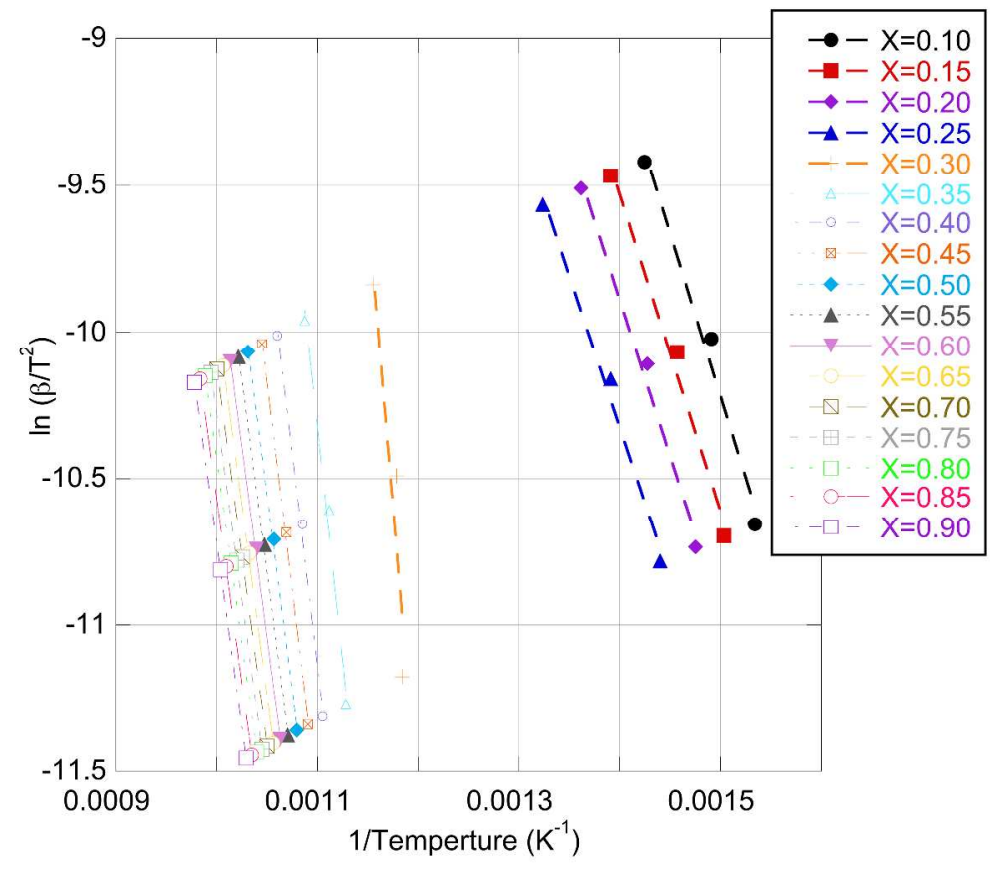

b. Pyrolyzed Semicoke 


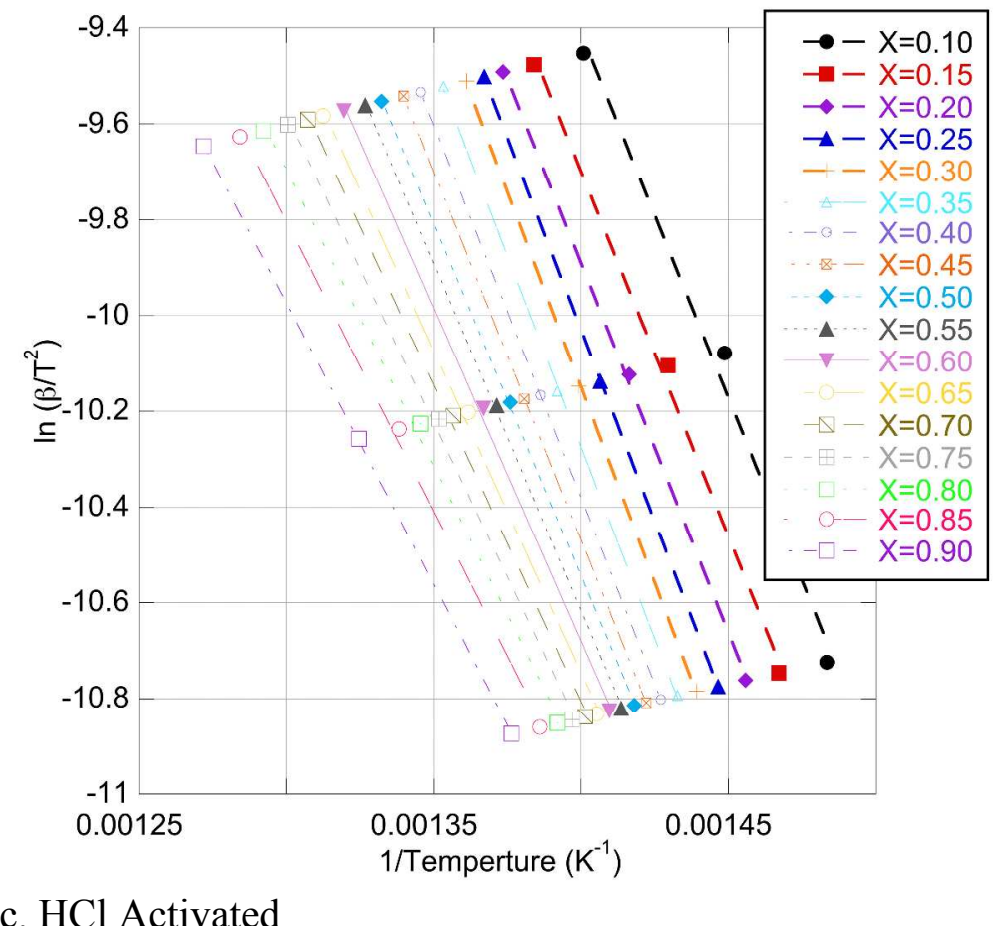

c. $\mathrm{HCl}$ Activated

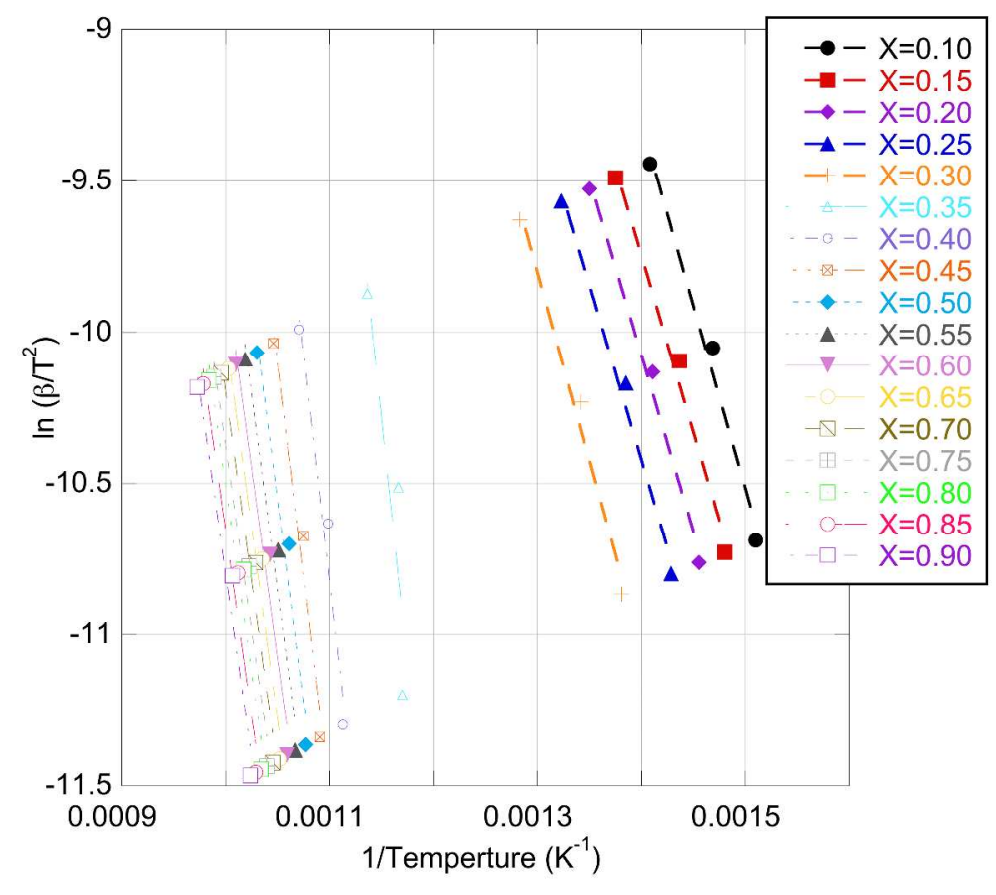

d. KOH Activated 


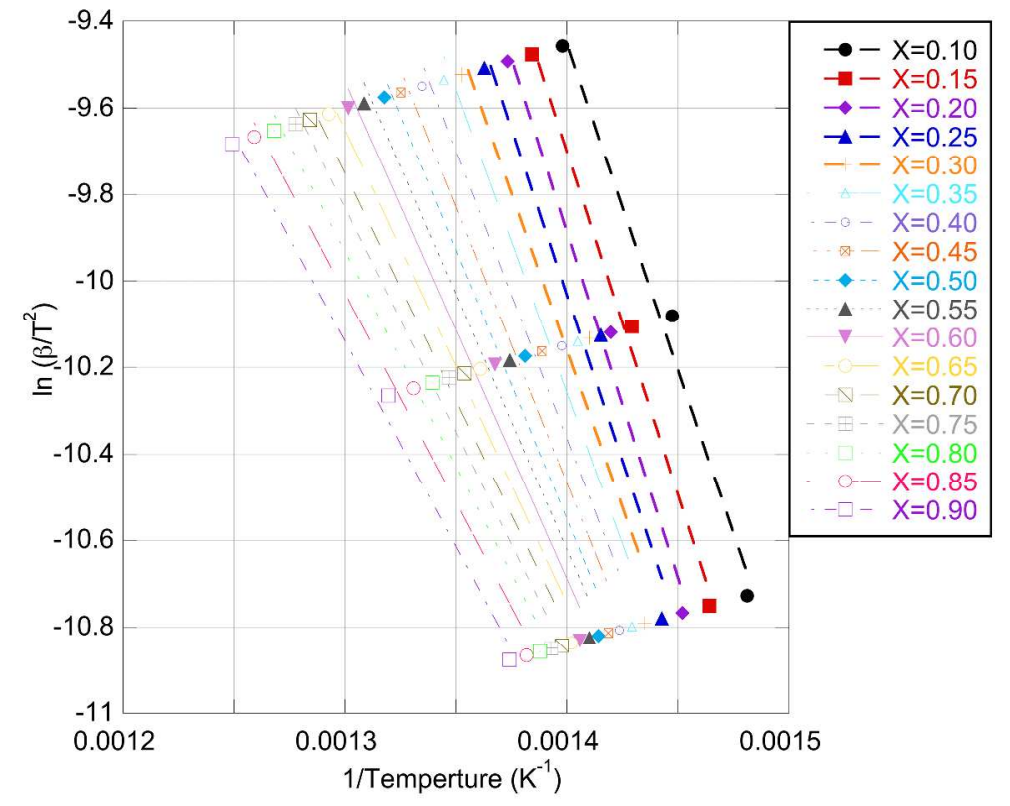

e. $\mathrm{HCl}+\mathrm{KOH}$ Activated

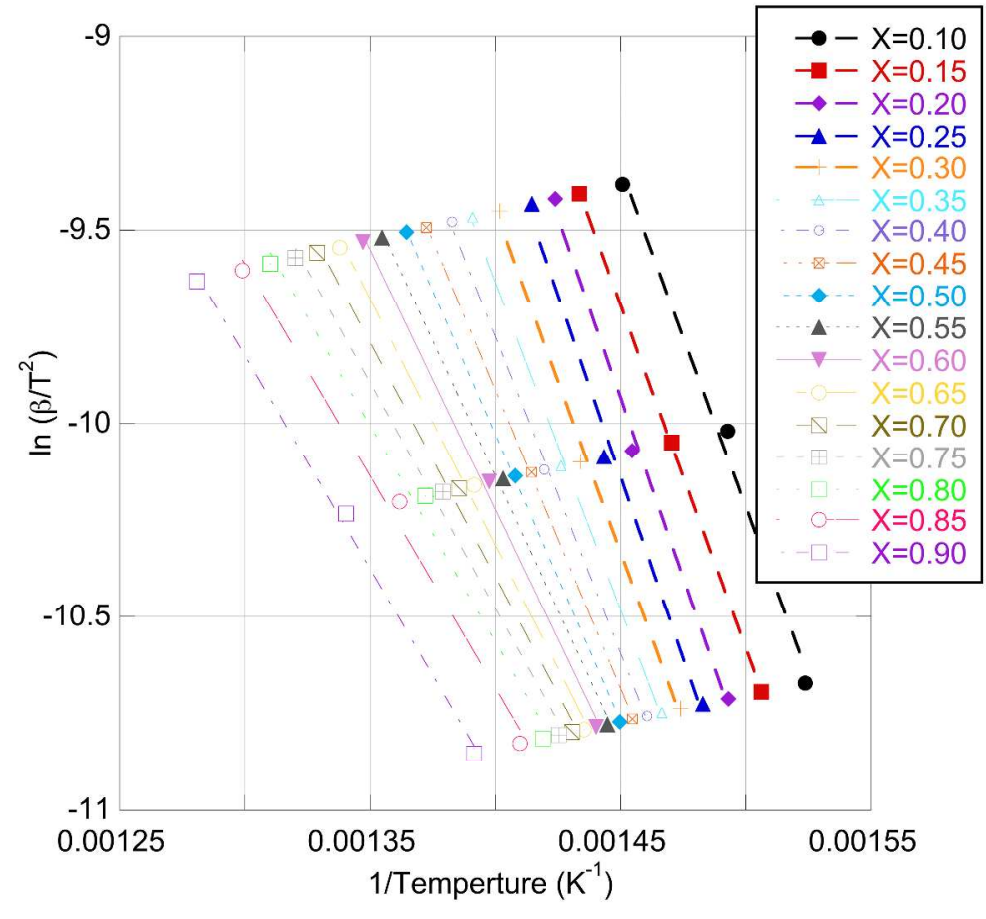

f. $\mathrm{KOH}+\mathrm{HCl}$ Activated

Figure S3. Isoconversional plots for oxidation of raw, semicoke and activated semicokes 